\title{
Neuronavigation in Reconstructive Surgery of a Large Skull Defect after Decompressive Craniectomy: Case Report
}

\author{
Zhanna Semenova, MD, PhD* and Andrei Marshintsev \\ Research Institute for Emergency Pediatric Surgery and Traumatology, Moscow, Russia

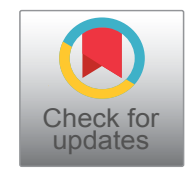

*Corresponding author: Zhanna Semenova, MD, PhD, Research Institute for Emergency Pediatric Surgery and Traumatology, Moscow, Russia

\begin{abstract}
Introduction: The concept of reconstruction of skull defects is based on the need to restore anatomical relationships for the maximum possible recovery of brain function.

In the absence of autogenous bone due to various reasons, the choice of an implant becomes an important problem of reconstructive surgery. With introduction of computer modelling and development of prototyping technologies the issues of cosmetic reconstructive operations are becoming less relevant. In late cranioplasty implant production and installation is complicated by the presence of significant bone growth along the perimeter of cranial defect. The ability to adjust implant to a bone window with uneven edges is not always possible, even with personalized manufacturing.

Materials and methods: A case of late closure of skull defect after a decompressive craniectomy by an implant from RLS Martin PEEK material in an 8-year-old boy is presented. Our goal is to show possibility of optimizing late reconstructive surgery of complex skull defects using a preconditioned implant.

Results: There were no complications in the perioperative period. Borders of resection of bone outgrowths were marked according to presurgical navigation planning based on stereolithographic model of patient's skull. Control CT scans demonstrate almost complete alignment of stereolithographic skull model and performed reconstruction of cranial wall.

Conclusion: The use of neuronavigation made it possible to accurately outline the limits of resection of bone outgrowths to achieve complete congruence of bone window (defect) and the implant.
\end{abstract}

\section{Keywords}

Cranial defect, Reconstructive surgery, Neuronavigation, Children

\section{Introduction}

History of cranioplasty is very long. In 1668 Van Meekeren described a closure of a skull defect in a young Russian gentleman by a dog bone. However the implant was later removed under the threat of excommunication [1]. Despite such a long history of reconstructive surgery of the skull, many problems remain unresolved. Timing of surgery and choice of material for closure of skull defects are still debated [2-4]. The number of patients with large defects after decompressive craniotomy has increased. This results to the syndrome of trephined skull [4-7].

Reconstructive surgery of skull defects is an integral part of neurorehabilitation. Restoration of anatomical integrity of skull results into normalization of cerebral perfusion, brain protection, and eliminates a cosmetic defect $[1,8,9]$.

Autologous bone remains a golden standard for cranial defects closure despite many papers on shortcomings of its use. Allografts such as titanium, PMMA, hydroxyapatite etc. are widely used as an alternative to autotransplantation. Each of them has its own advantages and disadvantages $[2,4]$.

An implanted material should be safe, biocompatible and cosmetic. Cosmetic issues in reconstructive surgery are mostly solved due to development of computer modelling and prototyping technologies. In case of late cranioplasty manufacturing and insertion of an implant is complicated due to bone overgrowth at margins of a defect lacking support ability.

Citation: Semenova Z, Marshintsev A (2020) Neuronavigation in Reconstructive Surgery of a Large Skull Defect after Decompressive Craniectomy: Case Report. Neurosurg Cases Rev 3:042. doi. org/10.23937/2643-4474/1710042

Accepted: September 19, 2020; Published: September 21, 2020

Copyright: (c) 2020 Semenova Z, et al. This is an open-access article distributed under the terms of the Creative Commons Attribution License, which permits unrestricted use, distribution, and reproduction in any medium, provided the original author and source are credited. 
Available implants have different strength grades which complicates their shaping during surgery. It means that an implant might not be always adjusted to a bone window. Moreover it should not hinder further diagnostic investigations.

We present a case of late ( 3 years after head injury) cranial vault reconstruction after decompressive craniectomy.

\section{Case Presentation}

An 8-year-old child suffered closed head injury 3 years ago due to fall from height. Initially he was hospitalized at a municipal hospital at his place of residence. On admission he had GCS score 6 and anisocoria. CT scans revealed acute epidural hematoma $(40 \mathrm{ml})$ on the right and midline shift $7 \mathrm{~mm}$ with compression of cistern ambiens. Decompressive craniectomy and removal of epidural hematoma was performed. Posttraumatic hy- drocephalus developed after surgery. VP-shunting with medium pressure valve was performed 12 months after initial surgery. Tonic seizures without loss of consciousness started. Several anti-epileptic drugs were administered.

On admission to our clinic (Research Institute for Emergency Pediatric Surgery and Trauma) on neurological examination: Minimal conscious state plus, paresis of right III and VI cranial nerves, pseudobulbar syndrome and spastic tetraparesis predominantly on the left (Ashworth 3). Optic nerves are partly atrophic. EEG showed epileptic activity in left fronto-temporal region and bilaterally in frontal and anterior temporal regions. No seizures were recorded on video.

MRI scans (Figure 1) showed enlarged and asymmetrical lateral ventricles. Subarachnoid spaces are also enlarged. There is a catheter in anterior horn of the left

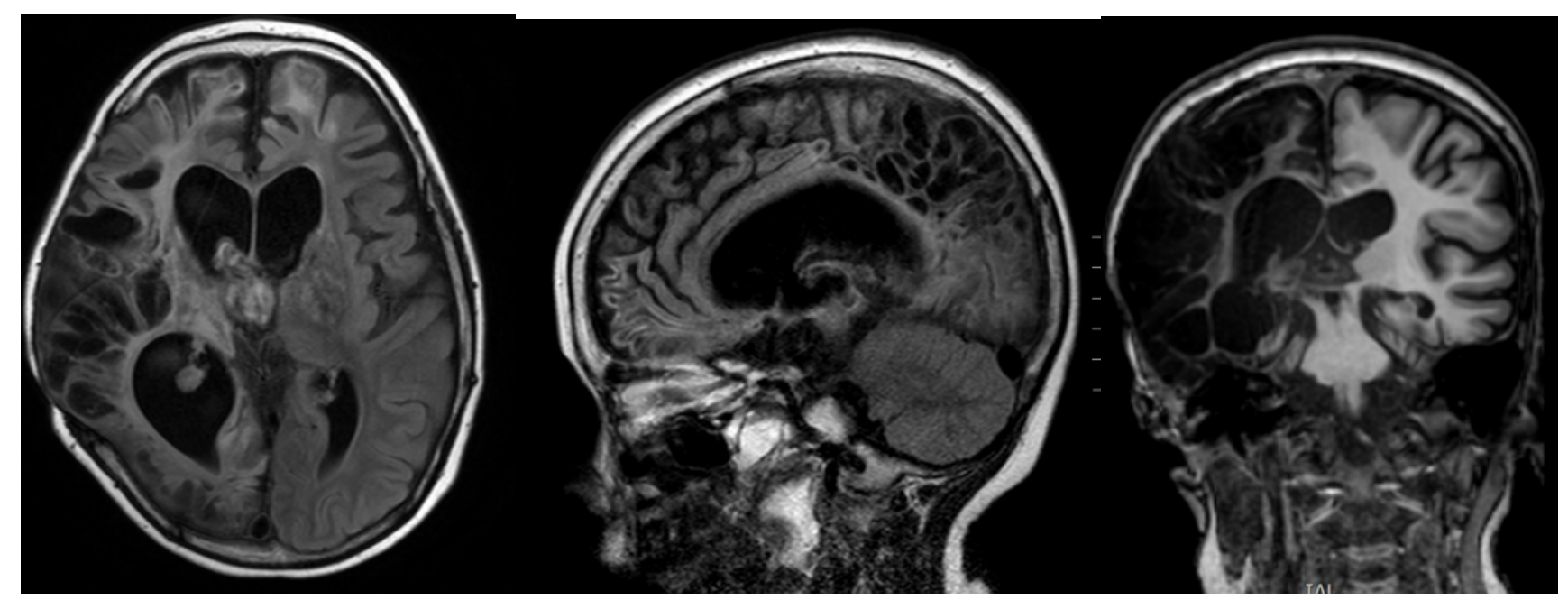

Figure 1: MRI 1, 5 year after injury. Multiple zones of gliosis mostly on the right side. CSF cavities are enlarged and deformed.
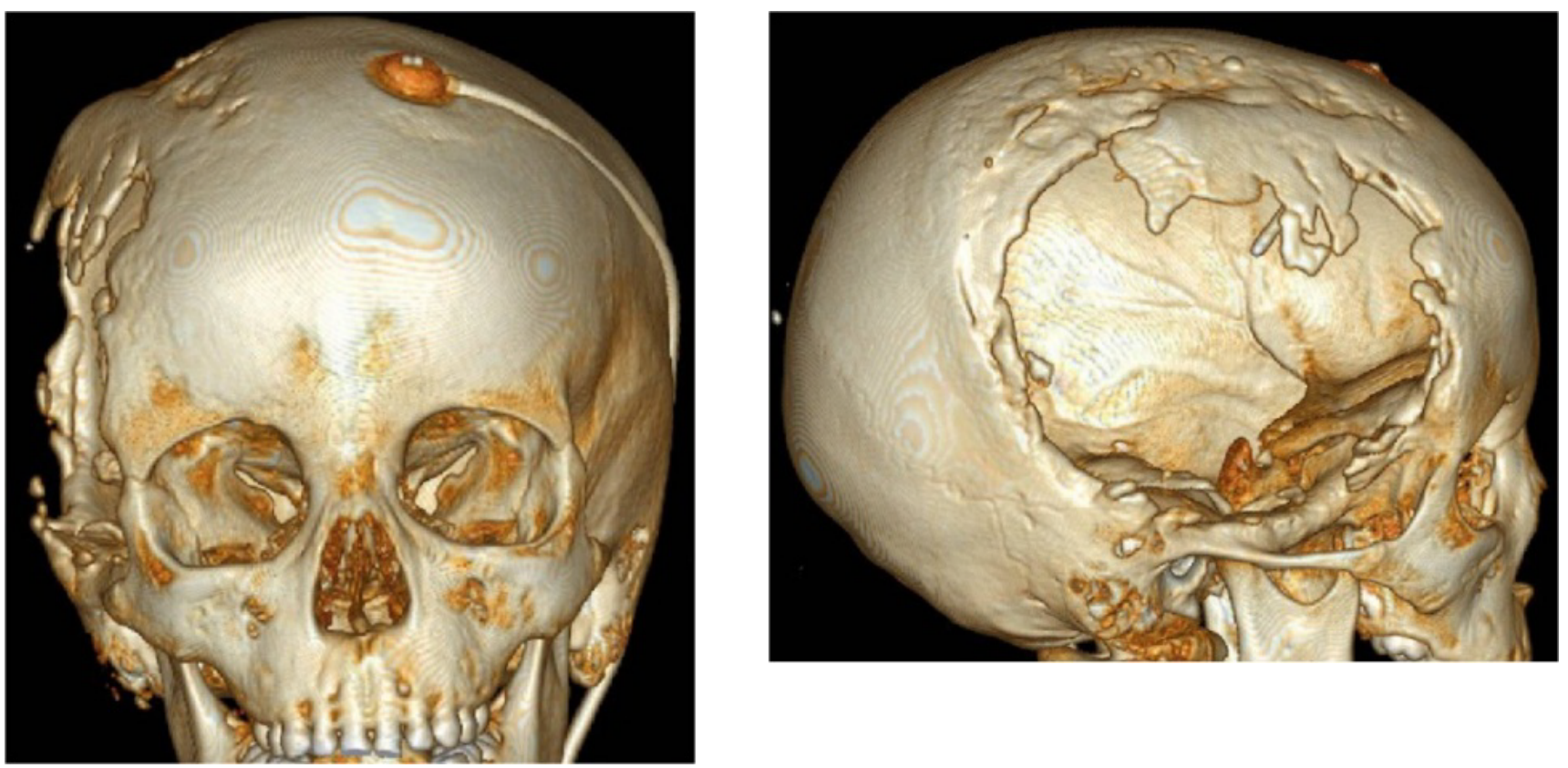

Figure 2: Cranial vault defect after craniectomy in right fronto-parieto-temporal region. 
ventricle. Small cysts and gliosis in the right hemisphere are visible. Corpus callosum is thin and heterodense. Basal nuclei are decreased. There are small multiple hypointensive signals in right parieto-temporal region. Basal cisterns are enlarged.

CT scans (Figure 2) showed extensive cranial vault defect in right fronto-parieto-temporal region. There are multiple foci of bone overgrowth at margins of the defect.

It was decided to close the defect by biopolymer synthetic implant PEEK (KLS Martin) produced with CAD/ CAM-technologies because it allows MRI scanning with minimal artifacts.

We performed 3D planning with electromagnetic system Asi EM of Medtronic Stells Navigation System. For this purpose CT-scanning of stereolithographic model of patient's skull was made. We superimposed images of 3D model of patient's skull and its stereolithographic model. Detailed contours of the post trepanation defect were marked. Resection of bone overgrowth was planned according to these marks (Figure 3).

Surgery was performed under general anesthesia. The child was in left lateral position. Skin incision fol- lowed the old scar. Marked adhesions betweens cart issues and dura mater were noticed. There were also numerous defects of dura filled by scary arachnoidea. Dural defects were repaired by periosteum and surgical sponge (Tachocomb ${ }^{\circledR}$ ).

Bone margins were separated from surrounding tissues. Borders of resection of bone outgrowths were marked according to presurgical navigation planning based on stereolithographic model of patient's skull. The outgrowths were resected using bone forceps and high speed surgical burr. Polymer implant was prepared. It was fixed with Y-shaped miniplates to the margins of the skull defect. Stages of surgery are shown on Figure 4.

Control CT scans demonstrate almost complete alignment of stereolithographic skull model and reconstruction of cranial wall.

Postoperative period was uneventful. Neurological examination after 6 months revealed vocalization and decrease of rate of epileptic seizures (GOS score 3).

\section{Discussion}

Indications for cranioplasty in children with skull defects are debated (patient's age, timing of surgery, and choice of implant), Size and localization of skull defect

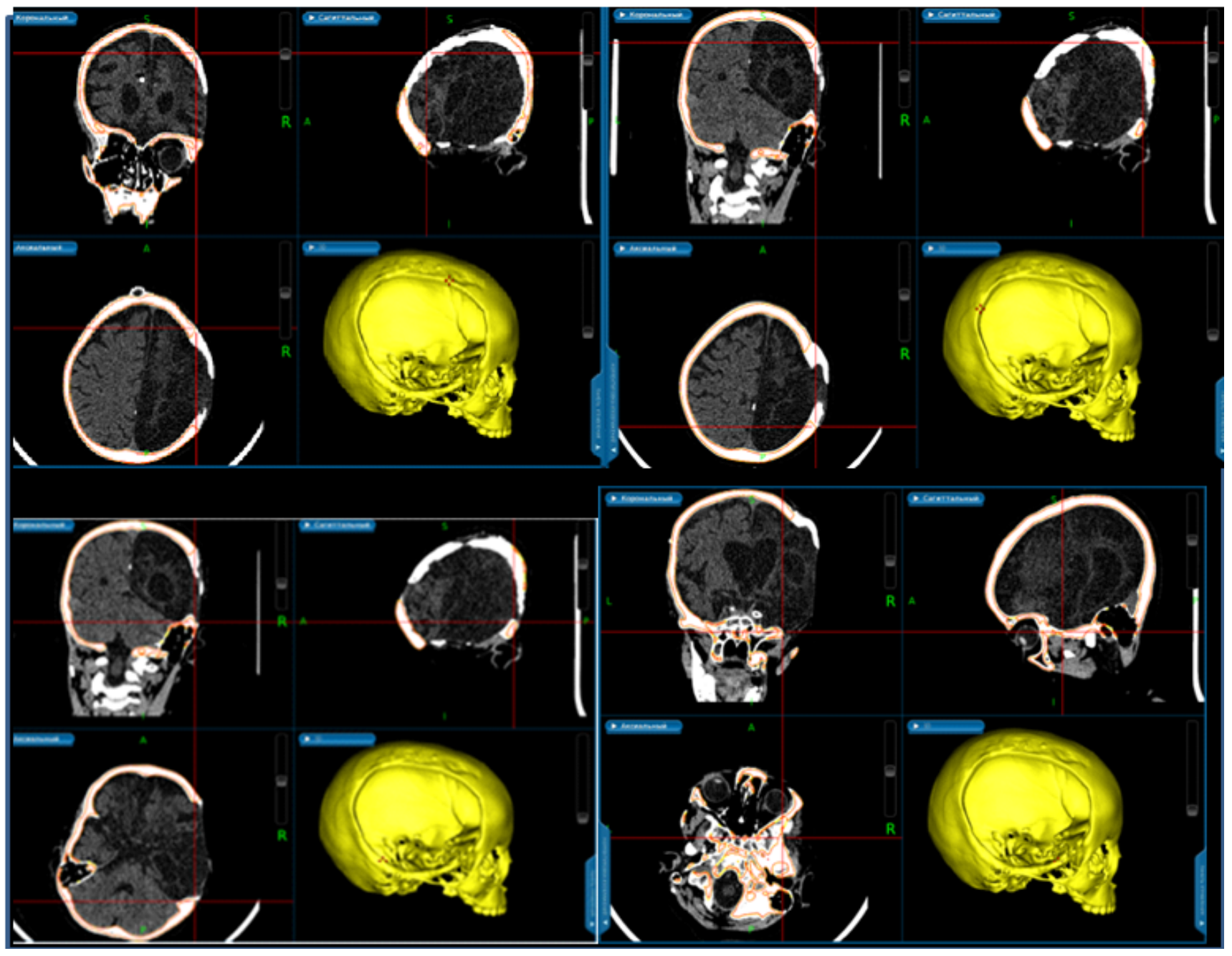

Figure 3: Presurgical planning with surgical navigation station. 


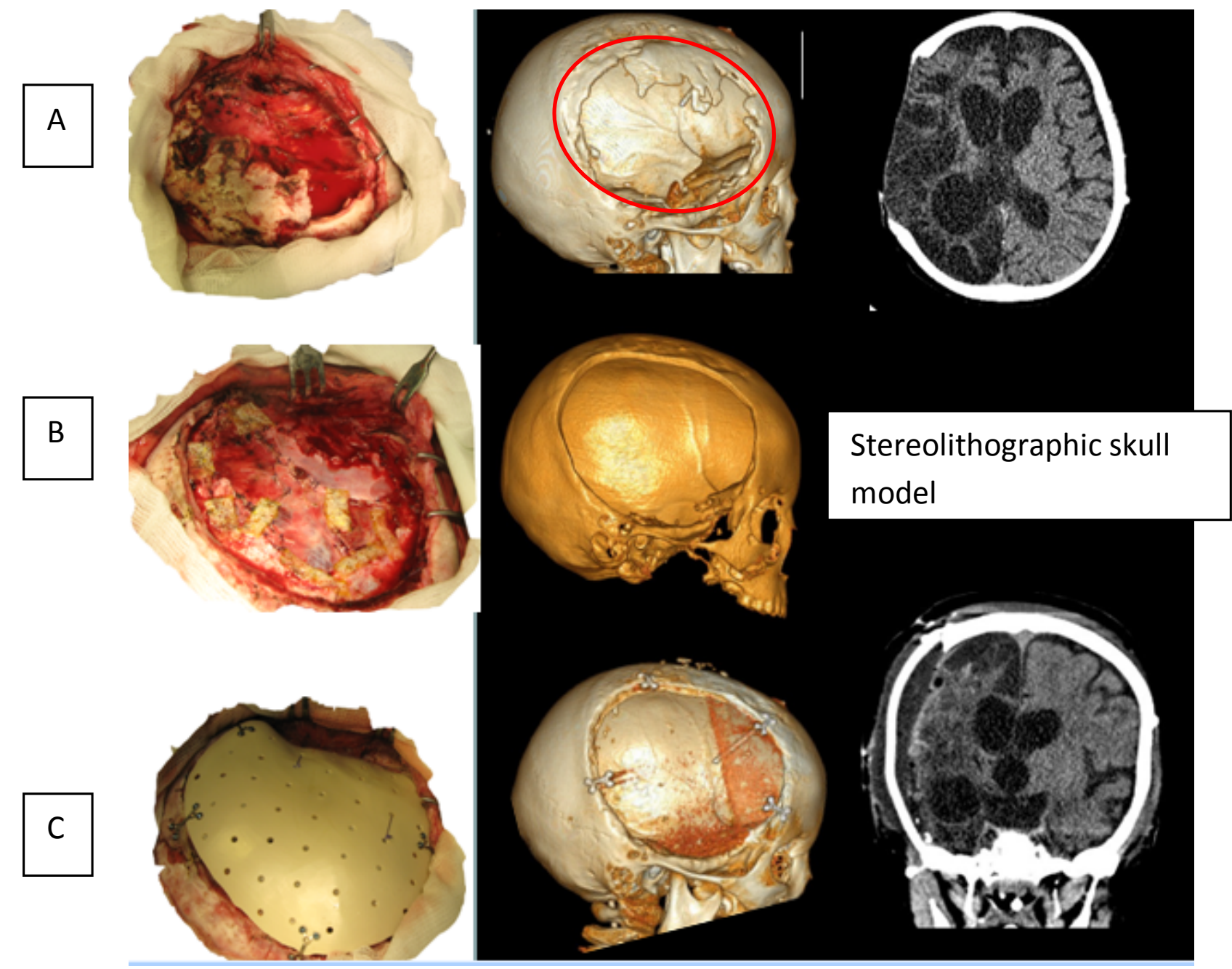

Figure 4: Stages of surgical intervention. (A) Forming of skin and scalp flap and delineation of margins of bone window; (B) Formation of bone window and duraplasty by periosteum and Tachocomb; (C) Implant fixation.

are considered main indications for surgery. The general trend is early closure within 1-6 months after injury $[5,6]$.

The primary goals of cranioplasty after DC are to protect the brain, achieve a natural appearance and prevent sinking skin flap syndrome (or syndrome of the trephined). Furthermore, restoring patients' functional outcome and closure of external defects helps patients to improve their self-esteem [9]. There is neurological improvement after cranioplasty that allows us to view this surgery as crucial part of neurorehabilitation after head injury in adults and children $[1,5]$.

The choice of closure material is one the main problems in cranioplasty. Autologous bone is preferred especially in children due to the skull growth. However is it not always possible (as in our case). Then we have to use allografts. These are foreign materials (-PMMA, titanium, hydroxoapatite, PEEK, etc.). We select an implant depending on patient's needs and possibilities. In our case we took into consideration the possibility of future MRI scanning. In addition, according to some data, in comparison of polyetheretherketone and titanium cranioplasty after decompressive craniectomy a trend toward increase in exposed implant in titanium cranio- plasty compared with PEEK cranioplasty was observed $(P=0.074)$ [9].

Manufacturing exact implant from different materials is possible by using different methods of 3D-prototyping based on CAD/CAM-technologies $[1,10]$. Such approach is widely practiced since early 1990s. However there are pathological bone outgrowths (especially in cases of late skull defects' reconstruction). That is why an implant is produced from a virtual bone defect with even borders but there is a problem of alignment between an implant and a bone defect.

Next requirement was maximal alignment of an implant and margins of a skull defect. Using high resolution spiral CT scans we produced a synthetic implant from PEEK and stereolithographic model of patient's skull taking in consideration detailed borders of the bone window. There are few publications on navigation for controlling reposition of bones after craniofacial injury [9-13].

Previously frameless navigation was used for surgical treatment of orbital posttraumatic defects and deformations. In such cases an implant was produced during surgery under neuronavigation control [8]. 
In our case we took an opposite approach by forming skull defect according to a preconditioned implant (KLS Martin PEEK manufactured with CAD/CAM technology).

PEEK implants are very durable and cannot be corrected intraoperatively. In our case the main problem was pathological bone outgrowths at margins of the skull defect. That is why we had to mark a virtual contour of the defect within normal bone before surgery. During surgery bone outgrowths were resected according to our plan. The use of neuronavigation allowed us to perform optimal volume of resection. It is very important, because excessive removal of bone tissue might result into incongruence between the size of an implant and a bone window. In such case implant fixation is difficult and it may sink into cranial cavity. The use of frameless navigation in such case is original solution of the problem.

\section{Conclusions}

Our case shows possibilities of neuronavigation in reconstructive skull surgery which might be recommended in similar cases.

\section{Conflict of Interest}

The authors have no commercial or financial associations that might create a conflict of interest with the information presented in this manuscript.

\section{Statement of Equal Authors Contribution}

All authors have contributed equally to the work.

\section{References}

1. Konovalov AN, Potapov AA, Likhterman LB, Korniyenko VN, Kravchuk AD, et al. (2012) Rekonstruktivnaya i miniinvazivnaya khirurgiya posledstviy cherepno-mozgovoy travmy.

2. Franco Servadei, Corrado laccarino (2015) The therapeutic cranioplasty still needs an ideal material and surgical timing. World Neurosurg 83: 133-135.

3. Hao Xu, Chaoshi Niu, Xianming Fu, Wanhai Ding, Shiying
Ling, et al. (2015) Early cranioplasty vs. late cranioplasty for the treatment of cranial defect: A systematic review. Clin Neurol Neurosurg 136: 33-40.

4. Thien A, King NK, Ang BT, Wang E, Ng I (2015) Comparison of polyetheretherketone and titanium cranioplasty after decompressive craniectomy. World Neurosurg 83: 176180.

5. Dujovny M, Evenhouse R, Anger C, Charbel F, Sadler L, et al. (1998) Preformed prosthesis from computed tomography data. Calvarial and dural reconsraction. In: Rengachary S, Benzel E, Neurosurgical topics. AANS Publ Com.

6. Patrick Schuss, Hartmut Vatter, Gerhard Marquardt, Lioba Imohl, Christian T, et al. (2012) Cranioplasty after decompressive craniectomy: The effect of timing on postoperative complications. J Neurotrauma 29: 1090-1095.

7. Stephens FL, Mossop CM, Bell RS, Tigno T Jr, Rosner MK, et al. (2010) Cranioplasty complications following wartime decompressive craniectomy. Neurosurg Focus.

8. Levchenko OV, Shalumov AZ, Farafontov AV (2009) Ispol'zovaniye bezramnoy navi-gatsii dlya plastiki obshirnogo defekta kostey lobno-glaznichnoy oblasti . Ney-rokhirurgiya.

9. Yong Jun Cho, Suk Hyung Kang (2017) Review of cranioplasty after decompressive craniectomy. Korean J Neurotrauma 13: 9-14.

10. Joffe JM, McDermott PJ, Linney AD, Mosse CA, Harris M (1992) Computer-generated titanium cranioplasty: Report of a new technique for repairing skull defects. $\mathrm{Br} \mathrm{J}$ Neurosurg 6: 343-350.

11. Cutting C, Grason G, McCarthy JG, C Thorne, D Khorramabadi, et al. (1998) A virtual reality system for bone fragment positioning in craniofacial surgical procedures. Plast Reconstr Surg 102: 2436-2443.

12. Metzger MC, Hohlweg-Majert B, Schun R, Matthias Teschner, Nils-Claudius Gellrich, et al. (2007) Verification of clinical precision after computer-aided reconstruction in craniomaxillofacial surgery. Oral Surg Oral Med Oral Pathol Oral Radiol Endod 104: e1-e10.

13. Matsuura H, Inoue T, Ogasawara K, Makoto Sasaki, Hiromu Konno, et al. (2005) Quantitavive analysis of magnetic resonance imaging susceptibility artifacts caused by neurosurgical biomaterials comparison of $0,5,1.5$, and 3.0 Tesla magnetic fields. Neurol Med Chir (Tokyo) 45: 395399. 